\title{
Learning Objects: Using Language Structures to Understand the Transition from Affordance Systems to Intelligent Systems
}

\author{
Jacques du Plessis \\ University of Wisconsin - Milwaukee, USA
}

jacques@sois.uwm.edu

\begin{abstract}
There has, of recent, been a marked interest in the utilization of learning objects, specifically on the potential of granularity and reusability to make instructional development more efficient, and learning experiences more customized and meaningful . Juxtaposition of affordance systems and intelligent systems reveals powerful possibilities for granularity and reusability. Biology, architecture and language are investigated as systems to explore the role of granularity and reusability, in the quest to better define current perceptions of learning objects in the realm of design and technology in instruction.

The notion of subsystems, and the rules that apply within each system (intra-systemic rules) and between subsystems (inter-systemic rules) as it extends to holistic purposes, defines the role of granularity. The inverse relationship between granular reusability and learning-objective specificity is addressed.

In conclusion, learning objects within the virtual context are evaluated to identify commonalities and patterns to inform the design and development of future learning objects, and to clarify granularity and how it impacts the scale and scope of reusability.
\end{abstract}

Keywords: learning objects, affordance, intelligence, artificial intelligence, granularity, reusability.

\section{Introduction}

This paper investigates the concepts of granularity and reusability as functional components of learning objects, in order to better classify learning objects into a logical taxonomy. Differences between knowledge objects and learning objects are considered beyond the scope of this paper, and are not addressed.

Material published as part of this journal, either on-line or in print, is copyrighted by the publisher of the Interdisciplinary Journal of Knowledge and Learning Objects. Permission to make digital or paper copy of part or all of these works for personal or classroom use is granted without fee provided that the copies are not made or distributed for profit or commercial advantage AND that copies 1) bear this notice in full and 2) give the full citation on the first page. It is permissible to abstract these works so long as credit is given. To copy in all other cases or to republish or to post on a server or to redistribute to lists requires specific permission and payment of a fee. Contact Publisher@ijklo.org to request redistribution permission.
Within the domain of reusability and granularity, affordance systems and intelligent systems are juxtaposed to project the future focus and direction of learning objects. An elaboration of reusability at different granular levels informs the way we think about the future development of learning objects. Within complex systems the focus shifts from the macro or system level to the subsystemic level. The building blocks at each subsystemic 
level exist to support how these building blocks communicate at the intra and inter levels, and how this impacts the range of final objectives. A key goal of this paper is to highlight subdefinitions of learning objects, and to suggest new terminology which provides better precision about what specifically is intended and not intended, as we communicate about learning objects. This should expedite the grounding of a common vocabulary, and a more precise sharing of universal terminology among practitioners and theorists.

Examples in the next section clarify the difference between affordance systems and intelligent systems. The examples used will also demonstrate the relative newness of intelligent systems as important and emerging innovations. The underlying technicalities in generating real-time natural language are discussed to illustrate the workings of an intelligent system. The technical generation of language is suggested as a frame of reference to shape the development of the learning objects of the future.

\section{Affordance Systems versus Intelligent Systems}

According to Turvey (1992) an affordance is a property of the environment. Norman (2002) states that

the word affordance was originally invented by the perceptual psychologist J. J. Gibson $(1977,1979)$ to refer to the actionable properties between the world and an actor (a person or animal). To Gibson, affordances are a relationship. They are a part of nature: they do not have to be visible, known, or desirable. Some affordances are yet to be discovered. Some are dangerous. I suspect that none of us know all the affordances of even everyday objects.

Thus an affordance addresses identifiable properties of objects, usually identified for the purpose of problem solving. An affordance system assembles the constituent parts with the intent to deliver the affordance or affordances of each part. This serves the holistic purpose of the system. A car, for instance, has many parts that interact to deliver the ultimate affordance of mobility.

Defining intelligence is controversial with many plausible definitions. For the purpose of the discourse in this paper, intelligence is defined as the ability to communicate - to receive data, to see the information therein, and to deliver a response based on the interpretation of the information according to a holistic frame of reference and purpose.

Historically humans provided the intelligence to all the affordance systems they created. The exception was the use of animals for their natural intelligence, in doing specific tasks like smelling, hearing, seeing, and other instinctual tasks. There are different realms and levels of intelligence. For example, different animals express intelligence differently. Each living organism has a particular capability to receive data, and to see the information within the data. For example, bats and moths use sonar detection of objects. Other animals use optical data to detect objects. The organism then uses the received information to perceive purpose within a holistic frame of reference, and then to respond accordingly. The bat, for example, uses sonar for the holistic purpose of detecting the moth. According to Metzner (1999), the tiger moth receives information and then uses a sonar response to create alternative echoes to avoid falling prey. This is a good example of receiving data, seeing the information therein, then responding appropriately, according to a holistic objective - survival in this case.

Given a competent driver, a car as an affordance system becomes an intelligent system. With the integration of intelligence, real or artificial, into a system, an affordance system becomes an intelligent system. The storage, transmission, logical interpretation and manipulation of digital data have opened up an historically new frontier of synthetic or artificial intelligence (AI) in which inanimate objects are imbued with decision-making ability. This ability continues to expand in 
sophistication. The following report exemplifies the transfer of intelligence to artificial environments.

The BBC online news posted the following: "An unmanned Russian spaceship carrying food has docked with the International Space Station, where supplies had been running dangerously low" ("Vital Food," 2004). Figure 1 indicates the existence of affordance systems and intelligent systems throughout human recorded history, and beyond.
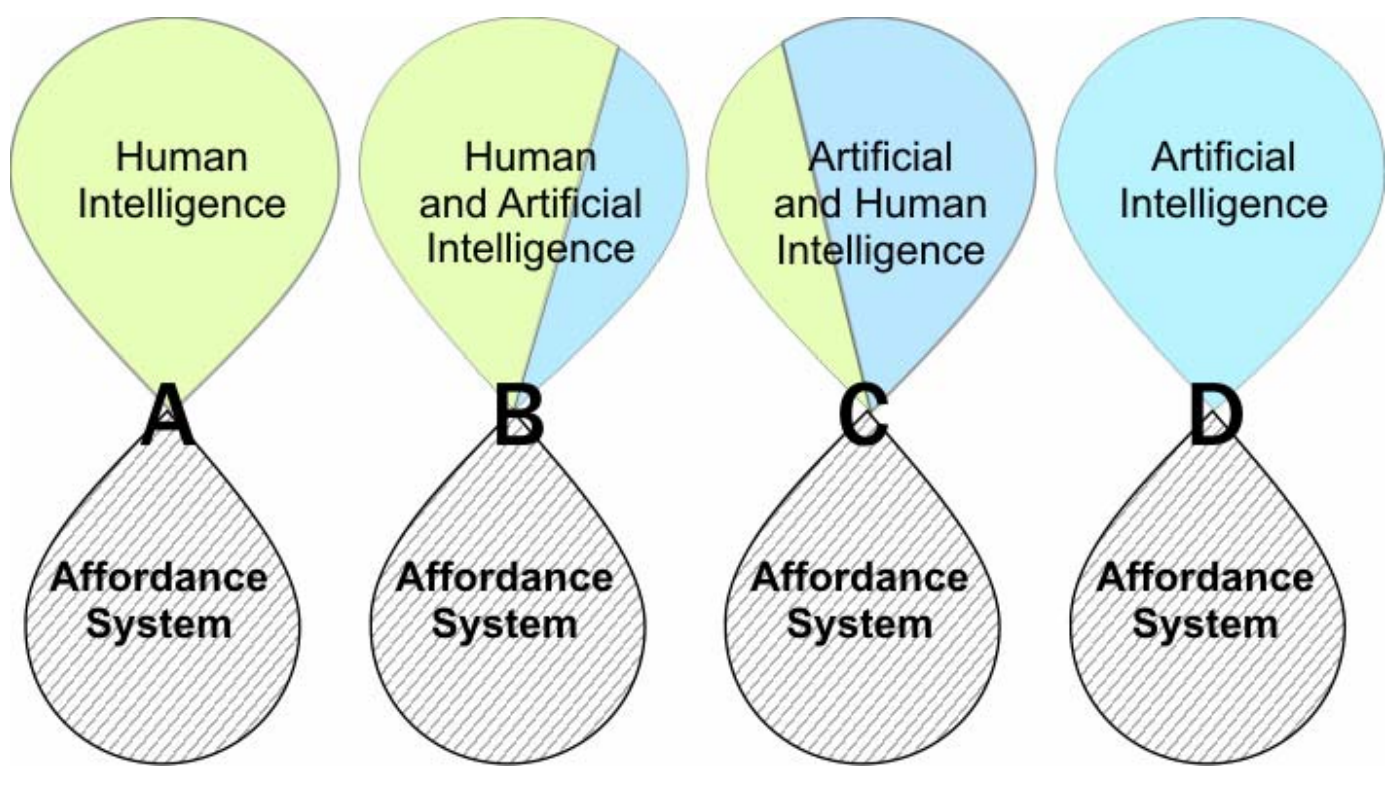

Figure 1: The expanding role of intelligent systems upon affordance systems

'A' indicates that throughout history, the only intelligent system possible was the combination of human or animal intelligence with the affordances of objects and systems. Juxtaposing an affordance system and an intelligent system in the traditional setting presents no new insights. The emergence of artificial intelligence increasingly enables traditional affordance systems to operate in specific situations with a reduced need or no need for human intervention, in order to achieve the holistic objective. This is a long-term projection for learning objects.

Situation 'B' is where limited intelligence is built into a system. A common example would be an outside light that has a motion sensor and light meter to provide light in the dark when needed. Its objective is to provide light while there is motion in low light conditions.

Situation ' $\mathrm{C}$ ' is an example where the role of AI has increased significantly. The Airbus A380 is an example of a sophisticated intelligent system (Adams, 2004). Within this specialized system, data are communicated to inform the system, being interpreted as just-in-time information about airspeed, altitude, weather conditions, etc. Because of the intelligence factor, this information is translated into in-flight action. Computer logic and analysis enables the craft to be transformed from an affordance system to an intelligent system. Another example, where the system operates with significant AI interventions and little human intervention, is the welding robots in car manufacturing.

Situation 'D' represents systems that function completely on AI. Drones in the military would fall in this category. These unmanned aircraft complete their mission based on preprogrammed in- 
struction, and then relying on built-in intelligence and communication to accomplish the objective.

Sandhana (2002) reports about the vision of intelligent drones in the combat missions of the future:

"Going well beyond autopilot and preprogramming, he envisions swarms of unmanned, unattended and untethered drones on the ground, in the air and underwater. These machines would be capable of independently handling events in a hostile combat zone, such as surveillance, strike and even capture and detention. Aiming to create an adaptive, dynamic, self-healing network of drones, Moshfegh intends to rework the whole idea of military structure.”

Because of the continuous ambition for cutting-edge technology, we have even seen laser-guided bombs going down a chimney. Yet, those who do not have the ability to acquire such intelligent systems of warfare (situations B-D), have responded with situation A intelligent systems. By paying the ultimate price, a suicide bomber becomes an intelligent war system, albeit technologically deficient.

\section{Simplex to Complex}

A simplex object is where the object itself does not break down into subparts. A complex object or system is one which uses a single name to collectively represent the subsystems and parts. Even though it is seen as a single entity, it is not. It exists as an ensemble. The following examples illustrate both simplex and complex systems.

- A needle has no subsystem, it is a simplex system.

- A power drill is a complex system consisting of subsystems, including a grip, switches, casing, electric motor, etc.

- A Boeing 747 is a collective name for en ensemble of systems. If you were to strip the plane part by part, it would be difficult to say at what specific point the structure has lost the denotation as a Boeing 747.

- $\quad$ ABC-blocks and Lego® blocks are simplex systems.

- The human body is a complex system of subsystems and many granular layers, e.g. DNA, molecules, proteins, cells, and tissue types.

- $\quad$ ABC-blocks represent simplex systems. Compare ABC-blocks, Lincoln Logs ${ }^{\circledR}$, Lego® blocks, and the Meccano set. Each building system offers a basic component and a designed way in which these interact. ABC-blocks have flat surfaces of the same size, using gravity to hold them together. Using gravity, a simplistic vertical and horizontal expansion is possible, with no design to bridge or span any distance beyond the size of the blocks.

- $\quad$ Lincoln logs offer a slightly more sophisticated way for the components to interact. The interlocking design goes beyond gravity to keep them together, thus expanding capabilities in three-dimensional space.

- $\quad$ Lego blocks add a simple and effective attachment system, allowing for a good pressure adhesion between the two blocks. Like with the two previouslymentioned systems, the attachment is emphasized on the horizontal plane, which limits the design options. 
- Meccano components allow for flexibility in their shape and the attachment mechanism allows for construction in each of the three planes in threedimensional space. Comparing these different building systems shows that the means by which the components attach to each other and the diversity of component shapes expands the sophistication of the possibilities to which a generalpurpose system can be applied. The Meccano system's combination of flexibility, its ability to attach on different planar levels, and its ability to accommodate movement and structural strength, enables it to be the base system for a complex physical system. Compared to ABC-blocks, the Meccano system is much better suited to allow the integration of subsystems into the cohesive whole. It is able to bridge the gap from a simplex to a complex physical system.

\section{Affordance vs. Intelligence; Simplex vs. Complex}

A key point of simplex systems is that they cannot be intelligent. An intelligent system subsumes an affordance system, to deliver the intelligent system, and a complex system subsumes simplex systems as the building blocks of the complex system.

Simplex systems are affordance systems. A simplex system is a system in isolation. An intelligent system implies movement of data, as well as an integrated complex system. Thus the data in conjunction with the delivery system implies a complex system in order to execute the communication implicit to an intelligent system. Using this logic, it becomes apparent why a simplex system cannot be an intelligent system.

From the perspective of the learning objective, learning objects could be simplex, which would be an entity in isolation, like a digital picture or a sound file. Within the realm of an affordance system, simplex learning objects (SLOs) could be combined or grouped. Through database technology, a very efficient retrieval and storage system can be developed for rapid access and retrieval of SLOs. If only simplex objects are stored and retrieved, this environment would be part of a Simplex Affordance System (SAS).

\section{(a) Simplex affordance systems (SAS)}

It is a system consisting of subcomponents, not necessarily serving any objective at this granular level. Examples of physical simplex objects would be bricks, a rope, or a pin. A virtual example would be an eight-bit character.

\section{(b) Complex affordance systems (CAS)}

With the definition of each of these terms, a physical example of a CAS would be an automobile before the introduction of on-board computer systems. Likewise a house in the suburbs is also an example of a CAS. The classic Canon F-1 camera is an example of a physical CAS. This sophisticated collection of specialized affordances becomes an intelligent system when placed in the hands of the expert (intelligent) photographer.

An example of a virtual CAS would be a word processor. Like the F1 camera, a word processor offers a sophisticated collection of specialized affordances that becomes an intelligent system when placed in the hands of seasoned (intelligent) editor. A CAS can be either virtual or physical. Physical systems, with basic computer technology onboard, provide an integrated CAS. 


\section{(c) Complex intelligent systems (CIS)}

The mind is the ultimate example of a CIS. Through complex affordance systems (the senses), the mind performs phenomenally complex tasks to receive data, see the information therein, and deliver an appropriate response, based on a holistic objective. As two gladiators or wrestlers enter a confrontational situation, several times per second there is a flow of especially visual and tactile data to the mind, which is then transformed into information and processed to deliver a response that is sent to many parts of the body. After this, an appropriate response is executed.

Future direction should be to structure the sub-componential objects within a simplex learning object, to be accessible to and integrated within complex learning objects. This in turn meets the needs of complex intelligent systems.

\section{Language - the Data and Information of a Complex Intelligent System}

It should be said immediately that the human mind is the intelligent system and not the language. Because of human intelligence language came into being. Through language, humans convey intent. Language is the oldest reusable knowledge object system. Language consists of stratified interconnected layers of essential functionality. In order to produce fluent language, the speaker has to develop a required automaticity within each layer, knowing the role of the granular layer in the context of the whole, and at a specific instant, what is needed and having access to all the variables that could satisfy that need. Mostly through a subconscious simultaneous cohesion at each level, the speaker produces a unified whole. Intelligence drives the choices within each layer - perceiving the possible variables, determining each particular choice to direct the formation of each granular stratum in the formation of meaning.

\section{The Affordance of Each Granular Level in Language}

It is not the intent to explore the utmost possibility of each granular layer. The objective is to take a holistic look, an observation of the big picture, to work with the obvious and the evident, to uncover essential interactions and purposes inside each layer and between layers needed to produce this holistic objective of spoken language. The layers of interest are: phonemes (sounds), morphemes (words and word components), semantics (words and phrases), sentences, paragraphs, and pragmatics (discourse) (Scott, 2000). The discussion of each granular layer will point to its role in delivering intended meaning - the deliverable of intelligence.

\section{Sounds - The Granular Layer}

Spoken language is a collection of sounds. Each sound or phoneme has a selection of possible variations or allophones. This is the base granular level of spoken language. Phonemes are the fundamental building blocks used to construct entities with expanded meaning. Each language has a rule system to determine which sounds are phonemes and which are acceptable allophones, and which phonemes can be used in what order. A set of sounds that includes several clicks would be needed if we were to speak Xhosa or Khoi (the Bushman language). If we speak English, those same sounds are not phonemes, but mere sounds. These clicks are now used for effect - to entertain or enhance expression. In contrast, Khoi conveys essential meaning integrated into the morphology and semantics of the language. The question then is not which sounds could be produced biologically, but which language is being spoken. Then, the particular language determines the collection of phonemes needed. Furthermore it also determines the allowable allophones that are acceptable for each phoneme. The allophones allow for regional differences in pronunciation. 
Since $l$ and $r$ are not allophones of the same phoneme in English, a transposition causes confusion and misunderstanding. In Japanese although $l$ and $r$ are allophones, then interchanging them would not have the same effect.

\section{Sounds - The affordance}

Any speaker can produce a wide variety of sounds. Then, through intelligence and repetitive training, to the point of fluent automaticity - the speaker dynamically selects from this repertoire of sound the precise collection of sounds to put together comprehensible words for a specific language. The conjoining of sounds is predicated by rules for the amalgamation of each sound in the particular context of other sounds, which sometimes dictates a shift or change or even omission of a particular sound. This is much more than a mere mechanistic conjoining of phonemes.

As with every other granular layer, the producer of language has to be able to automate the production of the sequence of sounds, and, at will, intervene in the automated process to consciously and deliberately alter the standard pattern. Furthermore, because of intonation, emphasis, dialects, or a speech defect, a speaker might use an allophone of a given phoneme.

\section{Word components (morphemes) - The granular layer}

Often the next layer beyond sound is seen as the word level. Words could consist of the root only, or a compound with prefixes, infixes and suffixes. The awareness of these subcomponents in words becomes a necessary conscious step in the production process, essential for precision in speech.

\section{Word components (morphemes) - The affordance}

With morphology, it is the understanding of the impact of the root, the prefix, the infix, or the suffix that determines the result. Often the selection of these subcomponents is vital in expression, as in the word workaholic which is derived from alcoholic. Words and word subcomponents convey syntactic and semantic meaning to further contextualize the greater purpose of the overall communication. The following word variations illustrate the need for a grasp of morphology: convey, conveyed, conveying, conveyance, conveyable, etc. At this level, the affordance is not in the nuances of pronunciation or inflection, but in the choice of words or word subcomponents. As with sound, there is automaticity in production, as one or another alternative component is a deliberate conscious intervention in the quest to clarify intention.

The required sounds must be produced with spontaneous immediacy. The greater the ability with prefixes, suffixes, infixes and words, the better prepared the speaker is to address the next level of semantics.

\section{Words and phrases (semantics) - The granular layer}

There is not a perfect one-to-one relationship between words (verbs or adverbs or adjectives or nouns) and the concepts they represent. Words like word processor or safety-valve inspector are two or more words in English, but in a language such as Afrikaans it is one word. Words are like stepping stones in the pool of meaning. Often the precise word is available to describe the intended meaning, and at times, specifically as we compare meaning between languages, we discover a precise term in language $A$ necessitates a broader description in language $B$. 


\section{Words and phrases (semantics) - The affordance}

The intelligence in the selection of words and phrases like idiomatic expressions and sayings are not only related to the dictionary equivalent meaning of every term, but it also relates to syntax the word order and grammatical necessities to convey the meaning. It is therefore the spontaneous consideration of grammar and semantics that impacts either the automated or conscious selection of vocabulary.

\section{Sentences (syntax) - The granular layer}

The sentence is often the shortest unit of a complete thought. It commonly includes a verb, but a one-word response like maybe serves as a complete thought as well. A sentence could possibly be complex and contain several ideas that are juxtaposed or interrelated.

\section{Sentences (syntax) - The affordance}

The ability to arrive at the desired expression with the dynamic arrangement of words and phrases to express a sentence is miraculous. It is awesome to consider the combination of the granular layers to this point - the sound system, the morphemic system, semantics, and syntax all in concert to express with remarkable precision the thoughts of the mind, including complex interrelationships between thought variables that have to be satisfied. Once more, the ability to automate the concurrent delivery of each of these subsystems makes this feat particularly significant.

\section{Inflection - The granular layer}

With the composition of language, each granular level is integrated with the next; the phonemes are grouped into morphological units; the morphemes are grouped into semantic units; the semantic units are aligned with correct syntax to form sentences which are layered with a particular inflection and render a particular meaning. Some of the variables used are a tonal rising, a tonal falling, a steady pitch, or a flux of down then up, or up then down, loudness (soft, loud), and length (long, short, staccato).

\section{Inflection - The affordance}

A sentence for example is treated as a whole by the speaker to create an inflectional pattern to support or specify meaning. In tonal languages, the inflection determines the semantic value of a word. In nontonal languages, the inflection guides the meaning. Language expressed without inflection, equals a loss of the intensity of the meaning, unmarked meaning, or the intended meaning not being rendered at all.

\section{Paragraphs and discourse (pragmatics) - The granular layers}

This is a collection of thought, with a broad cohesion of its constituent parts, and varying in length, ranging from one sentence to many sentences grouped. Paragraphs and discourse expands, with its own rules of persuasion, repetition of thought, economy of expression, etc. to support the intent of the message.

\section{Paragraphs and discourse (pragmatics) - The affordance}

The rules that pertain to discourse assume mastery of the preceding layers, as well as the skills of the orator or author. Errors in any of the lower granular levels transfer to the whole. One incorrect 
sound can bleed through to express the wrong message, even with correct morphology, inflection, and syntax.

The more fundamental the granular level, the fewer units exist, and the smaller the reusable units are. The higher the granular level, the larger the set of clustered components might be. This means the precision of the meaning is more fixed, less pliable, and applicable only to a narrowed context. Reusability is thus reduced.

It is possible that the whole discourse is a paragraph. It is possible that a sentence is a whole paragraph. It is also true that a single word is a sentence. And finally it is theoretically possible that one sound is a word. This speaks of the overlapping and the minimal content of the broadest levels. On the other hand, the possibilities are open-ended.

\section{Vital Observations about Granularity and Reusability}

Within the context of each granular layer, reusability exists. The first thing to notice with language from a holistic perspective is that there is an inverse relationship between granular reusability and contextual applicability (i.e. the lower the granular layer (e.g. Phonetics), the more reusable the granules are, and the higher the granular layer (e.g. Discourse), the less reusable the granules are, the less uniform they are in size, and the more context specific they are). As indicated in Figure 2 below, with development, the objective is to develop at a granular level to maximize reusability.

The other observation about this continuum is that at the lower granular levels, there are relatively few granules and at the higher the granular levels, the number of possible granules expand exponentially. As one moves up from the phonemic layer to the top layer of discourse, meaning is extremely rudimentary at the phonemic level and very precise at the discourse level. The more fixed and clear the meaning becomes, the more limited the reusability becomes.

In the development of an artificial intelligent system for the manipulation of learning objects the ultimate objective is not set on avoiding the reality as described in the observations above, but rather to understand it and to exploit the rules of reusability at each granular level. As users of

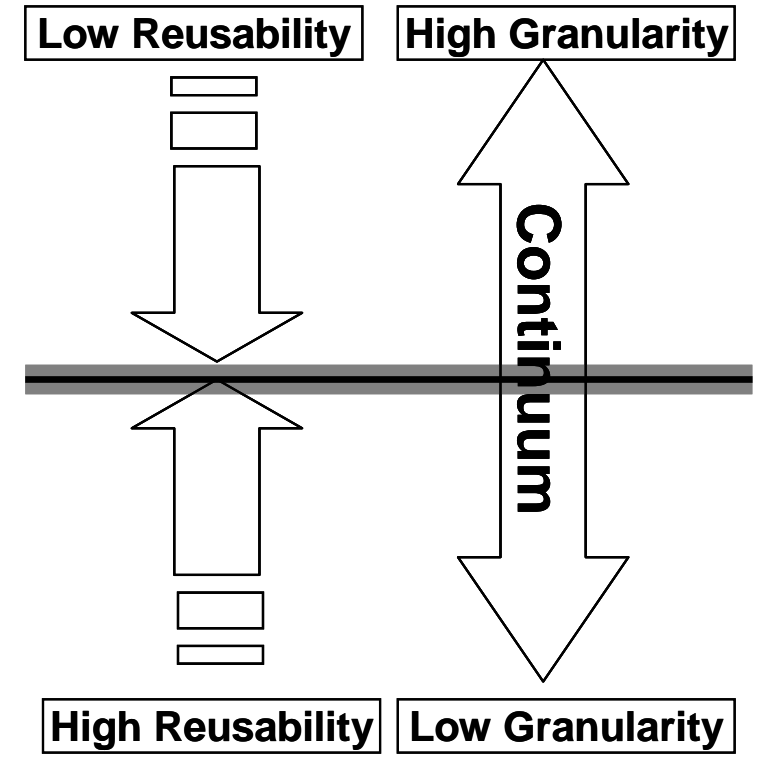

Figure 2: Satisfying the realities of reusability and granularity 
language we reflect that understanding from a very tender age.

\section{Digital Granularity and Reusability}

In the digital realm, binary bits are the fundamental layer. The next layer is bytes, which is the foundation for vectors, pixels or characters. Each path from bits to characters, vectors, or pixels respectively has different holistic destinies with the bytes reflecting different affordances. Bytes, pertaining to each of these three tracts, inform the greater whole in different ways, which might still change with the transition from 32- to 64-bit processors.

\section{Graphics}

For pixels, the intelligence ranges from a one bit pixel, rendering either black or white, to a 24-bit pixel with millions of colors. From the article "Bit Depth, Color Depth" at http://www.devx.com/projectcool/Article/19997/0/page/7 comes the illustration shown in Figure 3 of expanding the holistic possibilities of bits, ranging here from 1 to 24 bits ("Bit Depth," n.d.).

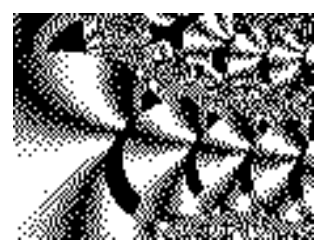

2 colors

1 bit

GIF

1,329 bytes

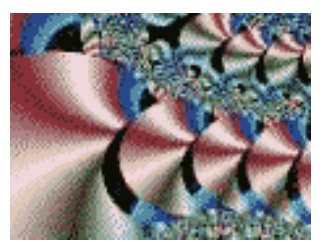

16 colors

4 bit

GIF

4,407 bytes

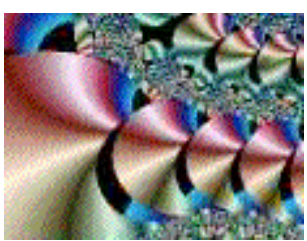

256 colors

8 bit

GIF

8,822 bytes

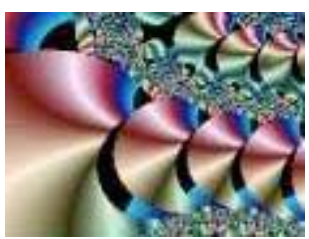

16 million colors

24 bit

JPEG

4,321 bytes

Figure 3: Rendering the same image as a 1, 4, 8, or 24 bit graphic.

\section{Text}

For text, the standard is still ASCII, evolving from the original 7-bit character set with only 128 characters to a byte (8-bit) character set with 256 characters. Unicode with the 16-bit character set can theoretically display 65,535 characters. An extension to this set (UCS-4) can display over a million unique characters. (http://codeworks.gnomedia.com/archives/2003/11/22/a-short-historyof-character-sets/)

Manipulating each type of object has a different rule set. Natural language and digital information have similar patterns. There is the same notion of granular levels, with high reusability and a limited set of options at the lower levels and at the higher levels, an infinite number of granules that differ in size. With language, the most basic independent granules are morphemes. Morphemes can be words, and thus deliver the essential purpose of language, which is meaning. Some phonemes are morphemes too, and would carry meaning.

With digital data, the essential purpose is a deliverable. A deliverable could be an ASCII file with one character in it. The bits are clustered into bytes. These bytes are inherently designed to become different types of objects, e.g. vector objects, bitmaps, character or sound, or bitmap files. Some with self-contained behaviors like animated GIFs. Objects have a particular meaning, just as morphemes have a collectively agreed meaning in language. The term index refers to an access system or infrastructure that enables a program to access precisely the object it needs. 
The option is, of course, for the program and the object or objects to be folded into one expanded object that is self contained as a deliverable. This option in terms of linguistic behavior would be the creation of communication, followed by capturing the expression to have on hand as needed. The alternative to this would be a rich repository of these different kinds of digital objects, with a layering of indexing, and a communicative programming language that is communicative. Based on what the learner required at that time, the program assembles the objects and proceeds with the learning transaction.

Thus we have two types of deliverables. The first would be a deliverable with fixed content and predetermined to achieve a given aim. This would operate within an affordance system. The other is a dynamic structure, like language, where the event will determine the objects to be accessed. This moves toward an intelligent system. Today's computer games present us with a low-level context-specific intelligence. There is an awareness of where the player is, and accordingly the communication between player and opponent happens.

\section{Conclusion}

In conclusion, these two paths of object manipulation: the affordance system approach and the intelligent system approach will always co-exist. The expansion of intelligent systems will completely depend on robust affordance systems to deliver on command. The indexing component in the form of metadata will provide access for intelligent systems to negotiate increasingly more complex decisions.

The future role of learning objects and their dynamic compilations will go beyond linear computational manipulations. At each granular layer manipulations will happen. An over-simplified possible illustration demonstrates this point: to express a sentence, the system would know the words it needs, and each word will trigger a search for its phonetic set. Feedback from the domains of semantics and syntax would determine that the plural noun needs a morphological adjustment and that the past tense also needs a morphological adjustment to the verb. This will retrigger another search for the appropriate phonemes. As these processes come together, the mood would be determined, and the inflection would trigger another search of the phonetic variation to express accurate intonation and inflection.

Based on the understanding of affordance systems and intelligent systems, we are in the transition from an information era to a communication era. Systems will be developed to fetch, render on demand, and also deliver responses based on interactivity. Like language, learning objects will utilize the metadata components, so that the expanding the repository of objects will be readied for communication. At different granular levels, subcomponents to learning objects will be understood and dynamically conjoined in learning objects. The quest is to achieve greater levels of automaticity. The dynamic creation of human speech serves as the model and archetype of automatic processing and dynamic compilation of objects. Future enhancements will constantly focus on the indexing capability to find the precise object (or subcomponent) when needed, and to programmatically perform manipulations to deliver within the holistic potential of the environment. The core of this quest is the same core quest of language. It is the ability to communicate. With learning objects it is specifically the ability to implement decision power to create and meet learning objectives.

\section{References}

Adams, C. (2004, December 28). A380 Innovations: A Balancing Act. Aviation Today. Retrieved February 2, 2005, from

http://www.aviationtoday.com/cgi/av/show_mag.cgi?pub=av\&mon=0303\&file=0303a380.htm. 
Bit Depth, Color Depth. (n.d.) Reference Guide: Graphics, Technical Options and Decisions. Retrieved February 4, 2005, from http://www.devx.com/projectcool/Article/19997/0/page/7

Metzner, J. (1999, July). Tiger moths: Cry. Retrieved February 2, 2005, from http://www.pulseplanet.com/archive/Jul99/1928.html

Norman, D.A. (2002) Affordances and design. Retrieved February 2, 2005, from http://www.jnd.org/dn.mss/affordances-and-design.html

Sandhana L. (2002). The drone armies are coming. Wired News. Retrieved February 2, 2005, from http://www.wired.com/news/technology/0,1282,54728,00.html

Scott, E. (2000). What is natural language? Retrieved February 2, 2005, from http://wwwrohan.sdsu.edu/ ling354/nat-lang.html

Turvey, M. (1992). Affordances and prospective control: An outline of the ontology. Ecological Psychology, 4 (3), 173-187.

Vital Food Reaches Space Station. (2004, December 26). BBC News - UK Edition. Retrieved February 2, 2005, from http://news.bbc.co.uk/1/hi/sci/tech/4122733.stm

\section{Bibliography}

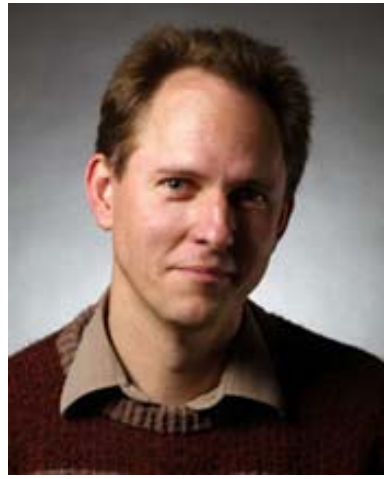

Jacques du Plessis has been active in the field of Instructional Technology since 1985. He did his PhD in Instructional Technology at Utah State University. His current research interests include human computer interaction, learning objects, and speech server technology. 